\title{
Underestimates of unintentional firearm fatalities: comparing Supplementary Homicide Report data with the National Vital Statistics System
}

\author{
C Barber, D Hemenway, J Hochstadt, D Azrael
}

Injury Prevention 2002;8:252-256

\begin{abstract}
Objective: A growing body of evidence suggests that the nation's vital statistics system undercounts unintentional firearm deaths that are not self inflicted. This issue was examined by comparing how unintentional firearm injuries identified in police Supplementary Homicide Report (SHR) data were coded in the National Vital Statistics System.

Methods: National Vital Statistics System data are based on death certificates and divide firearm fatalities into six subcategories: homicide, suicide, accident, legal intervention, war operations, and undetermined. SHRs are completed by local police departments as part of the FBI's Uniform Crime Reports program. The SHR divides homicides into two categories: "murder and non-negligent manslaughter" (type A) and "negligent manslaughter" (type B). Type B shooting deaths are those that are inflicted by another person and that a police investigation determined were inflicted unintentionally, as in a child killing a playmate after mistaking a gun for a toy. In 1997, the SHR classified 168 shooting victims this way. Using probabilistic matching, 140 of these victims were linked to their death certificate records.

Results: Among the 140 linked cases, $75 \%$ were recorded on the death certificate as homicides and only $23 \%$ as accidents.

Conclusion: Official data from the National Vital Statistics System almost certainly undercount firearm accidents when the victim is shot by another person.
\end{abstract}

n 1997, 981 people in the United States died from unintentionally inflicted firearm injuries according to the National Vital Statistics System of the Centers for Disease Control and Prevention (CDC), our nation's official death count. But is that number accurate?

The number is based on information that the medical examiner or coroner provides on the victim's death certificate about the injuries and conditions that contributed to the death, the mechanism by which the injuries were inflicted, and the manner of death: whether natural, homicide, suicide, accident, pending, or undetermined. Death certificates are processed at vital statistics registries where they are assigned a cause of death code using the International Classification of Diseases (ICD) coding system. ${ }^{12}$ The broad coding categories for firearm injuries differ only slightly from the "manner of death" categories used by the coroner or medical examiner and include homicide, suicide, accident, legal intervention, war operations, and undetermined.

Many firearm injuries fall into a gray area for manner of death. In the mid-1990s in a series of articles entitled "Mind Your Manners", medical examiners Hanzlick and Goodin charted differences in how medical examiners code the manner in which a person dies. ${ }^{3}$ To illustrate their findings, they presented to 150 medical examiners attending a 1996 NAME meeting a death scene scenario in which a hunter mistakenly shot another hunter. Accident or homicide? By a show of hands, half chose accident, half chose homicide.

What explains the split in medical examiners' determination when the facts establishing the incident as unintentional were not in dispute? Under the law enforcement and dictionary definition, homicide is the killing of one person by another, whether intentionally or unintentionally. Using that definition, homicide and accident are not mutually exclusive categories. Although lay people often use "homicide" as if it were synonymous with "murder", from the law enforcement perspective the term encompasses murder, manslaughter, justifiable homicide, and the accidental killing of one person by another. Medical examiners who chose "homicide" for the manner of death in the hunting scenario were not inaccurate; they were applying one definition of the term.

Vital statistics registries, however, apply a different definition. Under the ICD, the system by which the United States and most countries assign official cause of death, the "homicide" category refers to fatal injuries "purposely inflicted by other persons". ${ }^{1}$ "Homicide" and "accident" are mutually exclusive categories within the ICD cause of death coding system.

The result of this disjuncture in definitions may be that deaths which under the ICD coding system should be assigned to the "accident" category are instead assigned to the "homicide" category on the death certificate. Investigators with the University of Miami's Jackson Memorial Medical Center reviewed medical examiner reports on pediatric gunshot wound deaths in Miami-Dade County over a five year period. ${ }^{4}$ The investigators determined that 26 of the firearm deaths were unintentionally inflicted. Among those 26, only four were coded as "accident" for manner of death by the medical examiner. Most of the remainder were coded as "homicide".

The Massachusetts Weapon-Related Injury Surveillance System reported similar results. ${ }^{5}$ Among five deaths reported by emergency department personnel and by newspapers as unintentional shootings, four were recorded on the death certificate as "homicides", not "accidents". When asked about the cases, Dr Stanton Kessler, Associate Chief Medical Examiner for the Commonwealth of Massachusetts, explained, "If

Abbreviations: CDC, Centers for Disease Control and Prevention; ICD, International Classification of Diseases; NVDRS, National Violent Death Reporting System; SHR, Supplementary Homicide Report 
one person kills another person, we usually call it homicide and let the courts decide whether there was any wrongdoing" (personal communication, 7 July 1997).

Is the experience of these two states typical of what is happening across the nation? To explore this question, we linked two national databases-one covering police reported homicides (Supplementary Homicide Reports; SHRs) and the other covering death certificates (National Vital Statistics System) - to learn how gunshot wound deaths that the police determined were accidental homicides were coded on the death certificate. We also examined the demographic characteristics of the victims and perpetrators, the gun types, and the precipitating circumstances involved in these shootings.

SHRs have been used extensively as a data source to study murder. However, they have received little attention as a data source for unintentional killings. SHRs are filled out by local police departments as part of the FBI's voluntary Uniform Crime Reports system. Cases that are suspected to be murders, violence related manslaughters, law enforcement related killings, or homicides committed in self defense are reported as offense type A cases ("murder and non-negligent manslaughter"). Cases that are determined to be unintentional killings of one person by another (excluding motor vehicle crashes) are reported as offense type B cases ("manslaughter by negligence"). A bystander who is unintentionally killed by a stray bullet during an assault would be recorded as a type A homicide. A child killed when his or her playmate mistakes a gun for a toy would be recorded as a type B homicide.

Since 1984, reporting of negligent manslaughters has not been a required component of the Uniform Crime Reports program; reporting is left to the discretion of the reporting agency. FBI personnel caution that local police departments often under-report type B homicides. While SHR data are, therefore, likely to yield an underestimate of the total incidence of unintentional gunshot homicides, those cases that they do identify are likely to be true positives for unintentional injury because they have been investigated by a police officer and determined to fall in the type B category.

\section{METHODS}

We obtained victim based SHR data for 1997 from the public access data archive maintained by the Inter-university Consortium for Political and Social Research. (Data for 1998 were available but did not include type B homicides.) Multiple cause of death data based on 1997 death certificate information were obtained from the CDC's National Center for Health Statistics.

We present SHR data for type B firearm homicides concerning weapon type, relationship of victim and perpetrator, and type of incident.

Type B homicides (unintentional shootings) from the SHR were linked to the death certificate dataset to determine whether they were recorded as accidents or as homicides. The records from the National Vital Statistics System database from which we sought linking records were those with the following ICD-9 codes for underlying cause of death: accidents (E922, E922.0-922.3, E922.8, E922.9), suicides (E955, E955.0E955.4), homicides (E965, E965.0-E965.4), legal intervention (E970J), and undetermined (E985, E985.0-E985.4).

Because the two datasets do not have a unique record identifier in common, they cannot be linked directly. Therefore, we used the handful of variables that the two databases have in common to link the databases probabilistically. The small number of common variables, and the clustering of murder victims among young males in urban counties, make the SHR and vital statistics databases ill suited for broad scale probabilistic linkage. However, for infrequent victim types who are not so geographically and demographically clustered-including accidental gun homicide victims and very young victims-a probabilistic linkage of SHRs to death certificate records is feasible.
In addition to homicide type, SHRs capture the age, race, sex, and Hispanic ethnicity of the victim, the month of the offense (which usually, but not always, corresponds to the month in which the victim died), the unique code assigned to the investigating police agency (ORI code), the state and county of the investigating police agency (which usually, but not always, corresponds to the county in which the homicide occurred), the weapon type, the type of situation (for example, single victim/single offender, multiple victim/single offender, etc), and information about the offender and circumstances. Among these variables, the data from the National Vital Statistics System capture the age, race, sex, and Hispanic ethnicity of the victim, the month of death, the county of the victim's residence and the county in which the death occurred (which usually, but not always, corresponds to the county in which the homicide occurred) and the weapon type.

For the 168 accidental gun homicide victims reported on the SHR, we attempted to link the SHR record to its corresponding death certificate using a series of 10 passes in SAS, followed by manual review of all linked and unlinked cases. Records were grouped by state; SHR cases that were reported in one state were sought only among the vital statistics records for residents of that state or for deaths occurring in that state.

Pass one required a perfect match on victim's age, race, sex, ethnicity, ORI county/county of death, and month of offense/ month of death. Thirty six cases matched, and no matching cases were duplicates (that is, no SHR record matched more than one vital statistics record). The matching cases were set aside. Pass two required a perfect match with the exception of Hispanic ethnicity which was allowed to be missing in the SHR record. An additional 60 matches were identified and set aside. Again none were duplicates. Each successive pass identified no duplicate matches within the pass, and cases identified at each pass were set aside before proceeding to the next pass. The terms of each pass were not additive (with the exception of allowing ethnicity to be missing); for example, while victim's race could be missing on one pass and victim's age could be plus or minus one year on another pass, no pass allowed both conditions to be true. The eight passes (followed in parentheses by the number of new matches they yielded) were: month of death in the National Vital Statistics System was one month after the SHR month of offense (three matches); SHR victim's age was plus or minus one year of the vital statistics victim's age (14 matches); SHR ORI county was missing (five matches); SHR race was missing (0 matches); SHR ORI county matched the vital statistics county of residence rather than the vital statistics county of death (seven matches); SHR county matched neither the county of residence nor the county of death in the National Vital Statistics System (for example, a person lived in one county, was shot in another, and was airlifted to a third where they died at a trauma center) (seven matches); race mismatched (one match); and month mismatched (seven matches). In total, these eight passes netted 44 additional matches for an overall match rate of $83 \%$ ( 140 of 168 SHR cases).

For children age 14 and under who were reported on the SHR as either a type A (murder and non-negligent manslaughter) or type B (negligent manslaughter) firearm victim, we also attempted to link to matching records in the vital statistics dataset. The linking method was the same. Because young children are infrequent victims of homicide, we were able to link both type A and B homicides at a match rate (84\%) comparable to that achieved for all-age victims of type B homicides $(83 \%)$. The linkage allows us to evaluate for children not only what proportion of SHR identified accidents were reported on the National Vital Statistics System as assaults, but the converse: what proportion of SHR identified assault deaths were identified on the vital statistics system as accidents. 
Table 1 Victims of unintentional firearm homicides identified by SHRs, 1997

\begin{tabular}{ll}
\hline & No (\%) \\
\hline SHR victims & $168(100)$ \\
SHR victims with linking death certificate record & $140(83)$ \\
Underlying cause of death on linking death certificate record: & $105(75)$ \\
Assault by firearm (E965.0-E965.4) & $32(23)$ \\
Accident by firearm (E922.0-E922.9, excluding E922.4) & $3(2)$ \\
Undetermined (E985.0-E985.4) & $140(100)$ \\
Total linked SHR/death certificate records & \\
\hline
\end{tabular}

Table 2 Characteristics of victims and perpetrators of unintentional firearm homicides, SHRs, 1997

\begin{tabular}{lll}
\hline & $\begin{array}{l}\text { No }(\%) \text { victims } \\
(\mathrm{n}=168)\end{array}$ & $\begin{array}{l}\text { No (\%) perpetrators* } \\
(\mathrm{n}=168)\end{array}$ \\
\hline $\begin{array}{ll}\text { Age (years) } \\
\text { 0-9 }\end{array}$ & $17(10)$ & $6(4)$ \\
$10-19$ & $89(53)$ & $91(54)$ \\
$20-29$ & $28(17)$ & $33(20)$ \\
$30-39$ & $16(10)$ & $17(10)$ \\
$40-49$ & $11(7)$ & $11(7)$ \\
$50-59$ & $1(1)$ & $7(4)$ \\
$60-69$ & $3(2)$ & 0 \\
$70-79$ & $3(2)$ & 0 \\
$80+$ & 0 & 0 \\
Unknown & 0 & $3(2)$ \\
Gender & & $156(93)$ \\
Male & $126(75)$ & $11(7)$ \\
Female & $42(25)$ & $1(1)$ \\
Unknown & 0 & $113(67)$ \\
Race & $116(69)$ & $47(28)$ \\
White & $44(26)$ & $2(1)$ \\
Black & $3(2)$ & $4(2)$ \\
American Indian/Native Alaskan & $5(3)$ & $2(1)$ \\
Asian/Pacific Islander & 0 & \\
Unknown & & \\
\hline *In the six cases involving multiple perpetrators, the characteristics of the first listed perpetrator only are \\
included.
\end{tabular}

\section{RESULTS}

A total of 168 victims were recorded in SHR data for 1997 as having been shot and killed by another person accidentally or unintentionally. Of those, $140(83 \%)$ could be linked to a matching death certificate. Of these 140 linked cases, 75\% (105) were recorded in the death certificate database as homicides, $23 \%$ were coded as accidents, and $2 \%$ were recorded as undetermined deaths (table 1). The proportion coded as homicides did not vary significantly by region of the country; $78 \%$ of the unintentional shootings in the West, $76 \%$ in the Northeast/Midwest, and $72 \%$ in the South were coded as homicides. There were also no statistically significant or suggestive differences in the proportion of cases coded as homicides when comparing the SHR cases that matched "perfectly" to a death certificate record with those that matched under relaxed assumptions (data not shown).

Type B homicide victims in the SHR dataset $(n=168)$ were young. Sixty three per cent were under the age of 20 , and $89 \%$ were under age 40 (table 2). Three quarters were male and $69 \%$ were white. All were shot in single victim incidents. In $96 \%$ of the cases, one person was identified as having perpetrated the shooting; in a handful of cases $(n=6)$ more than one person was identified as handling the gun (not shown). The demographic characteristics of the shooters largely mirrored that of the victims, except that there were more male shooters, and fewer shooters under 10 years old. Fifty eight per cent of the shooters were under 20 years old.

The 981 fatalities classified by vital statistics as firearm accident victims include both victims whose injuries were self inflicted and those whose injuries were inflicted by another person. The ratio is unknown. Thirty one per cent of the accident victims in the vital statistics system data were under 20 years old.

For the 168 type B homicides, in $71 \%$ of the incidents a handgun was used in the shooting (table 3). While information on the type of gun was included in $97 \%$ of the SHR records, it was provided in only $32 \%$ of the matching deaths certificate records (not shown). Negligent handling of the firearm was the precipitating circumstance in $60 \%$ of the cases, and children playing with a gun in $31 \%$. Hunting and gun cleaning were responsible for $5 \%$ and $1 \%$ of the deaths respectively. Ninety five per cent of the victims knew the person who unintentionally shot them. Most frequently this was a friend $(35 \%)$, acquaintance $(26 \%)$, family member $(22 \%)$, or intimate partner (9\%).

A total of 274 children age 14 and under were recorded in SHR data for 1997 as having been shot and killed by another person, whether in a type A or type B homicide. Of those, 231 $(84 \%)$ could be linked to a matching death certificate. There was a high rate of agreement between SHRs and the National Vital Statistics System on the classification of type A (violence related) homicides. The vital statistics data assigned 186 of the 190 type A SHR cases (98\%) to the homicide category and only three to the accident category.

\section{DISCUSSION}

The first step in the public health approach to injury prevention is creating a systematic surveillance system that allows for understanding the extent and nature of the 
Table 3 Characteristics of incidents involving unintentional firearm homicides, SHRs, 1997

\begin{tabular}{ll}
\hline & No $(\%)(n=168)$ \\
\hline Weapon type & $120(71)$ \\
Handgun & $24(14)$ \\
Shotgun & $19(11)$ \\
Rifle & $5(3)$ \\
Other or unknown gun & $100(60)$ \\
Circumstance & $52(31)$ \\
Other negligent handling of gun which results in death of another & $8(5)$ \\
Children playing with gun & $6(4)$ \\
Victim shot in hunting accident & $2(1)$ \\
All other manslaughter by negligence & \\
Gun cleaning death (other than self inflicted) & $58(35)$ \\
Relationship of victim to perpetrator & $44(26)$ \\
Friend & $37(22)$ \\
Acquaintance & $15(9)$ \\
Family member (excluding spouse) & $8(5)$ \\
Intimate partner & $6(4)$ \\
Stranger &
\end{tabular}

problem. The data collected must be accurate and adhere to a common set of definitions, for data affect scientific results, public perceptions, policy, and policy evaluations.

The official source for data on the incidence each year of accidental firearm fatalities is the National Vital Statistics System. Many factors influence the accuracy of these data. Some factors may lead to overestimates. For example, in the first decade of the 20th century, many homicides were reported in the mortality statistics as accidental deaths. ${ }^{6}$ In addition, for religious, financial and even political reasons, medical examiners and coroners have sometimes been reluctant to certify suicide as the cause of death for self inflicted injury and may thus misclassify some suicides as accidents. ${ }^{7}$

This article investigates a bias that may lead to an underestimate of unintentional firearm fatalities in the vital statistics system. When the shooting is other inflicted rather than self inflicted, medical examiners/coroners may classify the death as a homicide, even though most injury experts would classify the death as unintentional. For example, newspapers carried accounts of a 12 year old boy who was shot in the head by a fellow sixth grader in New Bedford, Massachusetts in $1996 .{ }^{8}$ The boys were playing in the yard with a parent's semiautomatic pistol. They believed the gun was unloaded because its magazine had been removed, but a round of ammunition remained in the firing chamber. The facts of the case were not in dispute, and the shooting was considered an unintentional manslaughter, according to First Assistant District Attorney Renee Dupuis in Bristol County, the prosecutor who handled the case (personal communication, 12 October 2001). The boy's death certificate classified the case as a homicide, not an accident.

To investigate whether this classification problem is widespread, we compared the classification of individual deaths in the National Vital Statistics System with those from the SHRs. Although SHRs clearly under-report accidental homicides, those that they do report are likely to be truly unintentional or accidental because they were investigated by police and determined not to fall in the type A ("murder and non-negligent manslaughter") category.

Of the 168 type B ("negligent manslaughter") homicides reported in the SHRs for 1997, we were able to match 140 with the death certificate. Of these, $75 \%$ (105) were classified in the vital statistics dataset as assaults rather than accidents.

Our results indicate that the National Vital Statistics System data almost certainly underestimate the number of accidental firearm fatalities that occur each year, at least with respect to other inflicted shootings. National data are not cur- rently available on the proportion of firearm accidents that are self inflicted compared with "other" inflicted, so it is impossible to establish a more accurate estimate of the number of unintentional firearm deaths in the United States. Sixty two per cent of the SHR accidents that were coded as homicides in the vital statistics data were to children and teenagers. Moving these cases from the homicide category to the accident category in the vital statistics data would increase the number of unintentional firearm deaths among 0-19 year olds in 1997 by a minimum-since SHR reporting is incomplete-of $21 \%$.

We investigated the possibility that while some accidents were coded as homicides in the National Vital Statistics System data, the reverse might also be true (that is, some murders may be coded as accidents in the vital statistics data) perhaps leading to inflated estimates of the incidence of accidental firearm deaths. We could not test this hypothesis for type A and B victims of all ages, but we could for young victims ages 14 and under. We could not link victims of all ages because probabilistic linkage of SHRs and vital statistics fails when more than one victim is of the same age, race, ethnicity, and sex in any given month in the same city, something which happens frequently among murder victims who tend to be young adult males in large cities. The problem of demographically indistinguishable victims killed in the same month and same city occurs very infrequently among child victims. Among that group, we found that $98 \%$ of the type A homicides ("murders and non-negligent manslaughters") were correctly coded as homicides in the vital statistics data; only three out of 190 records were coded as accidents. However only $30 \%$ of the unintentional shootings (type B homicides) of children were coded in the vital statistics data as accidents and $70 \%$ were coded as homicides. The misclassification problem, therefore, appears to be limited to the unintentional shootings.

We were unable to investigate the hypothesis that suicides may be misclassified as accidents in the National Vital Statistics System database, nor were we able to investigate the opposite hypothesis, that some self inflicted accidents may be classified as suicides. SHRs do not capture self inflicted firearm injury, nor does any other national database.

What could be done to improve reporting on unintentional firearm injury deaths? The ICD underlying cause of death code is assigned by nosologists at vital statistics registries. If sufficient information is provided in the narrative portion of the death certificate to identify the shooting as unintentional, these coders have the authority to code a case as accidental even if the medical examiner or coroner has checked off "homicide" as the manner of death. But this appears to happen infrequently. 
Attempts should be made to standardize the protocols by which medical examiners and coroners classify manner of death, as Hanzlick and Goodin have suggested. ${ }^{3}$ This is a laudable goal but difficult to implement, given the decentralized nature of the nation's death investigation system. Vital statistics registry personnel will need to cooperate with medical examiners and coroners to establish clearer communications and protocols on this issue.

The CDC has plans to launch a National Violent Death Reporting System (NVDRS) starting with a few states in 2002. The purpose of the system is to collect and link information about suicides and homicides from multiple sources, including death certificates, medical examiner and coroner reports, and police records. Such a system, if it collects information on all firearm related deaths as well and applies a uniform protocol for classifying the deaths as accidents, violence related homicides, or suicides, would address the misclassification problem. By collecting detailed data on the circumstances leading to death and on the specific weapon type involved, the system would also provide much needed information to efforts to prevent unintentional firearm related deaths and to prevent suicides, domestic violence, child abuse, school violence, and other homicides more broadly. Work has been underway in the private sector to pilot test a proposed prototype for the NVDRS. ${ }^{910}$ The pilot system has been implemented in a dozen states and metropolitan areas around the country, indicating that nationwide implementation of the NVDRS may well prove feasible.

Our analysis of misclassification of unintentional gun deaths in the National Vital Statistics System database is limited by the fact that vital statistics and SHR data cannot be linked directly through individual identifiers. Probabilistic linkage identifies likely matching pairs of records, but the accuracy of the match is not confirmed. Some of what appears to be misclassification could, in fact, result when an SHR case is mistakenly linked to a death certificate for a different victim. The fact that we found no statistically significant or suggestive difference in the misclassification rate when comparing perfect matches with cases that matched under relaxed assumptions suggests, however, that the misclassification problem is a not an artifact of mismatching.

Our analysis is also limited to those deaths that were reported by police on the SHR. Because reporting of negligent manslaughter is an optional component of the SHR system, FBI personnel caution that an unknown, but likely high, proportion of these cases go unreported.

While the SHR system under-reports unintentional firearm deaths, the fact remains that $75 \%$ of the unintentional deaths that the system did report in 1997 were reported in the nation's National Vital Statistics System database as homicides and not as accidents. As a nation, we may be undercounting the burden of unintentional firearm deaths.

\section{Key points}

- Medical examiners and coroners may define "homicide" and "accident" somewhat differently than the vital statistics system in the United States.

- $75 \%$ of police reported unintentional shootings of one person by another were recorded on the victim's death certificate as "homicide," not "accident".

- Unintentional gun deaths, when inflicted by another person, appear to be seriously under-reported in the United States vital statistics data.

\section{ACKNOWLEDGEMENTS}

This research was supported in part with grants from the Annie E Casey Foundation; the Atlantic Philanthropies; the Center on Crime, Communities and Culture of the Open Society Institute; the David and Lucile Packard Foundation; the John D and Catherine T MacArthur Foundation; and the Joyce Foundation.

\section{Authors' affiliations}

C Barber, D Hemenway, J Hochstadt, D Azrael, Harvard Injury

Control Research Center, Harvard School of Public Health, Boston

\section{REFERENCES}

1 World Health Organization. Manual of the international statistical classification of diseases, injuries, and causes of death: based on the recommendations of the Ninth Revision Conference, 1975, and adopted by the Twenty-ninth World Health Assembly. Geneva: WHO, 1977.

2 World Health Organization. ICD-10: International statistical classification of diseases and related health problems: tenth revision / based on the recommendations of the Tenth Revision Conference, 1989 and adopted by the Forty-third World Health Assembly. Geneva: WHO, 1992.

3 Hanzlick R, Goodin J. Mind your manners. Part III: individual scenario results and discussion of the National Association of Medical Examiners Manner of Death Questionnaire, 1995. Am J Forensic Med Pathol 1997; 18:228-45

4 Schaechter J, Stepanian GA. Deliberating on "deliberate": medical examiner coding of pediatric gun deaths. Abstract. American Public Health Association Annual Meeting, 2001 (http://apha.confex.com/ apha/129am/techprogram/paper_24947.htm).

5 Barber CW, Ozonoff VV, Schuster M, et al. Massachusetts Weapon-Related Injury Surveillance System. Am J Prev Med 1998;15(3 suppl):57-66.

6 Eckberg DL. Estimates of early 20th century United States homicide rates: an econometric forecasting approach. Demography 1995; $32: 1-16$

7 O'Carroll PW, Rosenberg ML, Mercy JA. Suicide. In: Rosenberg ML Fenley MA, eds. Violence in America: a public health approach. New York: Oxford University Press, 1991.

8 Boyle M. 12-year-old shot, killed. The Standard Times 1996; March 16, section A: 1.

9 Azrael D, Barber C, Mercy J. Linking data to save lives: recent progress in establishing a National Violent Death Reporting System. Harv Health Policy Rev 2000;2:38-42.

10 Barber C, Hemenway D, Hargarten S, et al. A "call to arms" for a national surveillance system on firearm injuries [editorial]. Am J Public Health 2000;90:1191-3. 